\title{
Research Paper \\ The Effect of a Resistance Training Course on Blood Pressure and Nitric Oxide Levels in Elderly Women
}

\author{
Alireza Behjati Ardakani ${ }^{1}$, ${ }^{*}$ Ahmad Qassemian², Maryam Koushki², Elham Shakour², Ahmad Mehrez ${ }^{2}$
}

1. Department of Sport Sciences, Faculty of Literature and Humanities, Shahrekord University, shahrekord, Iran.

2. Department of Physical Education, School of Education \& Psychology, Shiraz University, Shiraz, Iran.

Received: 23 Aug. 2017 Accepted: 21 Jan. 2018

Key words: Nitric oxide, Blood pressure, Older women, Resistance exercise chtation: Behjati Ardakani A, Qassemian A, Koushki M, Shakour E, Mehrez A. [The Effect of a Resistance Training Course on Blood Pressure and Nitric Oxide Levels in Elderly Women (Persian)]. Iranian Journal of Ageing. 2018; 13(1):1627. https://doi.org/10.21859/SIJA.13.1.16

dol": https://doi.org/10.21859/SIJA.13.1.16

\section{A B STRACT}

Objectives Endothelial function is an important factor in the assessment of atherosclerosis, lipid deposition in the inner walls of the arteries, high blood pressure, and heart failure. Vascular endothelial cells play an important role in regulating vascular activities by producing substances such as nitric oxide to stimulate vessels.

Methods \& Materials The current study was attempted to find out the effect of resistance training on old women's plasma nitric oxide levels and blood pressure. Twenty-four postmenopausal women (age: $67.37 \pm 6.02, \mathrm{BMI}=26.87 \pm 4.16$, and $\mathrm{WHR}=0.92 \pm 0.4$ mean $\pm S D$ ) were selected objectively and divided into control $(n=12)$ and experimental $(n=12)$ groups randomly. Experimental group performed resistance training for eight weeks, three sessions per week with 40-65\% intensity. Study variables were measured and recorded before and after training program. Paired and independent sample t tests were used for data analysis. The significance level was lower than 0.05 .

Results The results revealed that resistance training had a significant effect on nitric oxide levels $(P=0.01)$ and blood pressure $(P=0.006, P=0.002)$ in old women.

Conclusion A period of resistance training with present study characteristics can reduce both systolic and diastolic hypotension and increase plasma nitric oxide levels, and therefore is recommended for prevention of cardiovascular diseases, atherosclerosis, and hypertension in old women.

\section{Extended Abstract}

\section{Objective}

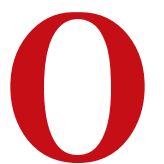

ne of the common diseases after menopause is atherosclerosis [1]. Vascular endothelial cells play an important role in regulating vascular activity by pro- ducing vasoactive substances such as nitric oxide [2] Nitric oxide acts as a vasodilator that can lower blood pressure with this mechanism [3,4]. Old blood vessels produce lesser amount of endothelial nitric oxide [5]. Furthermore, strong correlation reported between the reduction of nitric oxide bioavailability and the outbreak of cardiovascular diseases in postmenopausal women $[6,7]$. However, regular physical exercises, es-

\section{* Corresponding Author:}

Ahmad Qassemian, PhD Candidate

Address: Department of Physical Education, Faculty of Education and Psychology, Shiraz University, Shiraz, Iran.

Tel: +98 (937) 5852913

E-mail: ahmadqassemian@gmail.com 
pecially resistance exercises may reduce or delay the impairment of the endothelial function in the elderly people and causes the endothelial function to return in people with atherosclerosis [8, 9]. Therefore, due to limited research on the effect of physical activity on elderly people and the all the above-mentioned materials, current study was undertaken with the aim to investigate the effect of a resistance training course on the concentration of nitric oxide in elderly women.

\section{Methods and Materials}

This research was quasi-experimental and was performed as a 2-group research project with pre-test and post-test. 24 elderly women were selected purposefully as the research sample. They did not have any history of participation in resistance training and were not afflicted with heart disease or specific illnesses. After filling out the consent form by the subjects, they were randomly divided into two groups: resistance training group and control group $(n=12)$. First, the questionnaire of general health, physical activity level, and the medical history was completed by the subjects. To assess their initial situation, we measured their height, weight, BMI, daily systolic and diastolic blood pressure, and resting heart rate. Experimental group participated in course comprising eight weeks of resistance training, three sessions a week with 40-65\% intensity and at most one repetition [10-12].

Blood pressure was measured 48 hours before and after resistance training and $5 \mathrm{cc}$ blood sample was taken from each subject to measure the concentration of nitric oxide. Blood sampling was done at 8 a.m. and after 12 hours of fasting. Blood samples were taken, and immediately sent to the lab before starting the resistance training program. Blood plasma was separated with centrifuge and frozen at $-70^{\circ} \mathrm{C}$. After taking post-test blood samples and separation of blood plasma, samples were sent to the medical diagnostic laboratory at once for analysis. In the lab, concentration of nitric oxide was measured using the GLASY ELISA kit, USA, with a precision of $0.1 \mu \mathrm{mol} / \mathrm{L}$. Correlated t-test was used to review intragroup variations and independent t-test was used for inter-group differences. The level of significance was less than 0.05 . This research was approved by the Ethics Committee of the Physical Education Department of Shahrekord University.

\section{Result}

This study was conducted with a sample of 24 postmenopausal women [mean age of $67.37 \pm 6.02$, Body
Mass Index (BMI) of 26.87 \pm 4.16 , and Weight-Hip Ratio (WHR) of $0.92 \pm 0.4$ who were selected purposefully and divided randomly into experimental and control groups. There was a significant no significant difference between the two groups in terms of the general characteristics of the subjects and the mean variables of the research before intervention. However, it should be noted that average weight and average WHR of experimental group decreased after intervention and those differences were significant at the level of $\mathrm{P} \leq 0.05$.

Based on the findings, it has been determined that mean Nitric Oxide (NO) levels in the experimental group in the pretest and posttest setting were 22.58 and 27.00, respectively, and the difference between them was significant in this group $(\mathrm{P}=0.008)$. In the control group, there was no significant difference between the means of NO in pre-test and post-test scores, which were 25.50 and 24.50 , respectively $(\mathrm{P}>0.05)$. Independent t-test results showed that there was a significant difference between the means of NO in the post-test in both groups $(\mathrm{P}=0.01)$. In other words, intervention of independent variable (resistance training) had significant effect on the increase in NO levels in the experimental group. Therefore, it can be said that eight weeks of resistance training significantly increases NO levels in elderly women (Figure 1).

Our study also confirmed that systolic $(\mathrm{P}=0.006)$ and diastolic blood $(\mathrm{P}=0.002)$ pressure decreased significantly in the experimental group after the intervention, and based on t-test results on the average blood pressure in pre- and post-test. However, the mean difference between these two pressures was not significant in the control group in pre- and post-test $(\mathrm{P}>0.05)$.

In examining the relationship between variables, correlation ( $\mathrm{r}$ ) of nitric oxide concentration variable

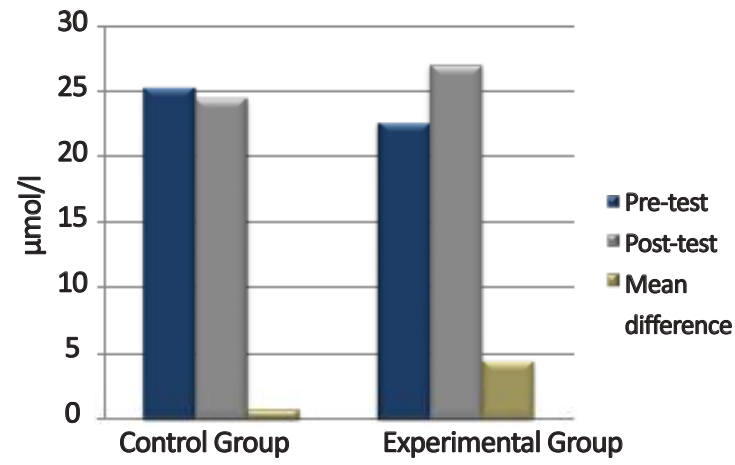

Iranian Journat of

Figure 1. Comparison of mean NO levels in control and experimental groups before and after intervention 
and systolic blood pressure equaled to 0.582 and the relationship was significant at the level of $\mathrm{P}=0.014$. Therefore, it can be concluded that there is a direct relationship between increasing plasma nitric oxide concentration and the decreasing systolic blood pressure. On the contrary, correlation of nitric oxide concentration with diastolic blood pressure found to be 0.257 , which was not significant at the level of $\mathrm{P} \leq 0.05$. In summary, the results of this study have shown that a course on resistance training has a significant effect on the increase in plasma nitric oxide levels and the decrease in the systolic and diastolic blood pressure in elderly women.

\section{Conclusion}

Our study established that a course of eight weeks on resistance training with $40-65 \%$ intensity and at most one repetition has a significant effect on the increase in plasma concentration of nitric oxide in elderly women and this is an important indicator to prevent cardiovascular disease, especially atherosclerosis and hypertension. Moreover, this training course caused a significant decrease in systolic and diastolic blood pressure in elderly women. Decreased blood pressure can be due to the reduction in catecholamines production, because of the training and the subsequently reduced environmental resistance. In addition, sports activities can lower blood pressure by increasing the number of capillaries in active skeletal muscle, reducing vascular resistance due to the diffusion, reducing blood flow resistance, improving nervous system regulation of blood vessels, and decreasing heart rate during rest and activity [13]. Considering the results of our study as well as other benefits of physical activity, resistance exercise can be recommended to the elderly and those who suffer from conditions like hypertension.

\section{Acknowledgments}

This research did not receive any specific grant from funding agencies in the public, commercial, or not-forprofit sectors.

\section{Conflict of Interest}

The authors declared no conflicts of interest. 


\title{
تأثير يك دوره تمرين مقاومتى بر فشار خون و نيتريكاكسايد در زنان سالمند
}

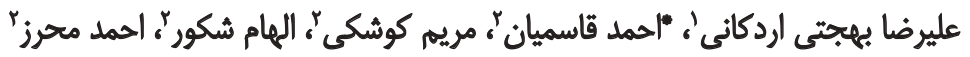 \\ 1- كروه علوم ورزشى، دانشكده ادبيات و علوم انسانى، دانشكاه شهركرد، شهركرد، ايران.

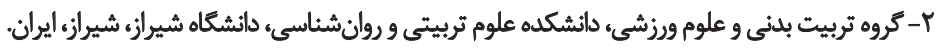

\begin{abstract}
حكيe

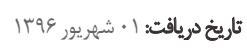

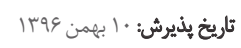

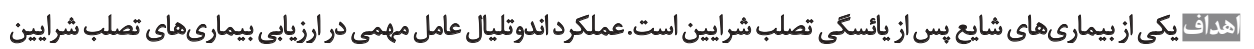

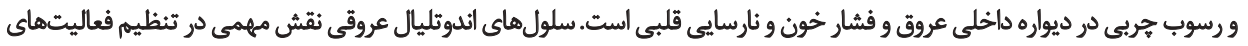

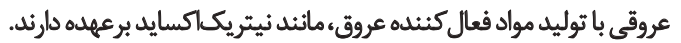

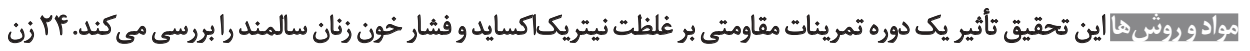

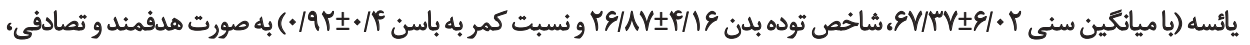

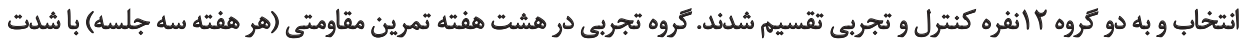

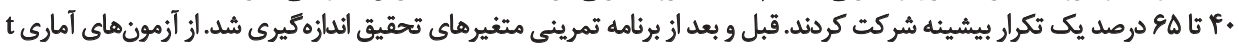

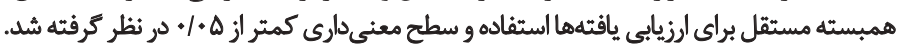

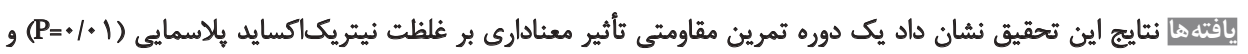

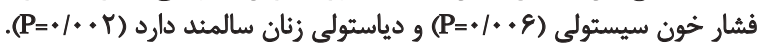

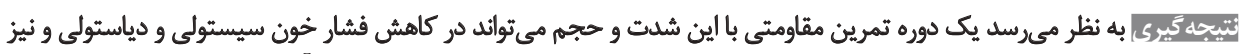

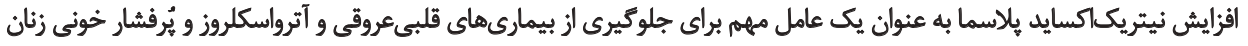
سالمند مؤثر باشد.

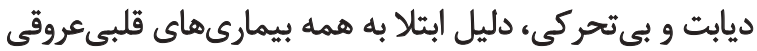

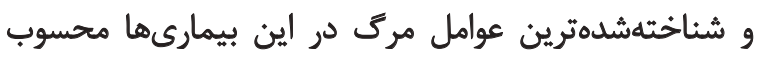

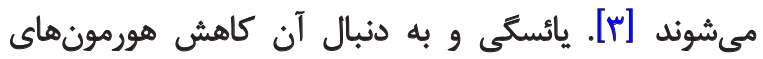

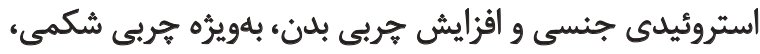

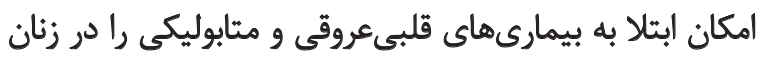

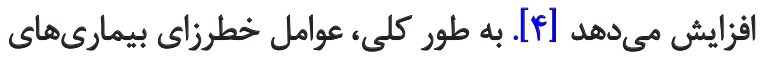

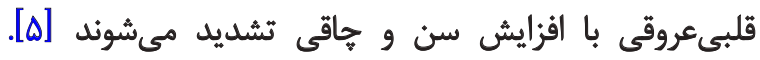

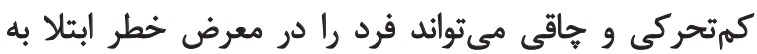

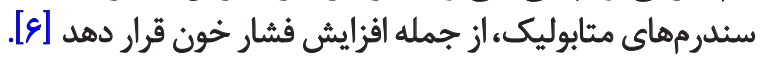

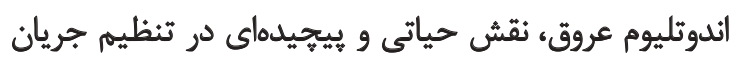

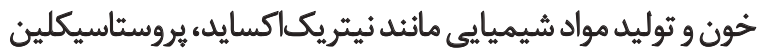

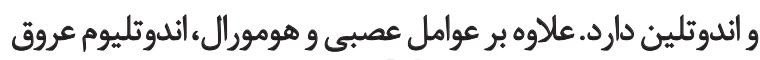

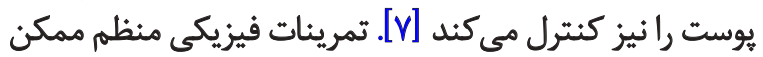

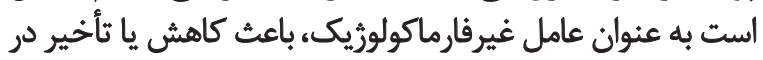

dateo

يكى از جدى ترين مشكلاتى كه جامعه جهانى رانكران كرده،

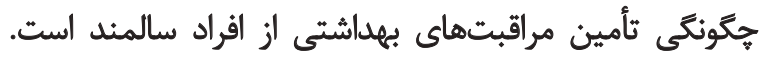

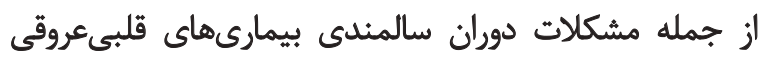

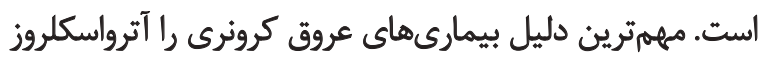

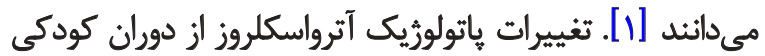

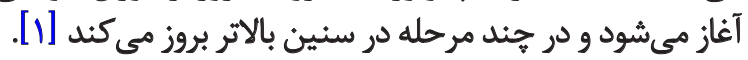

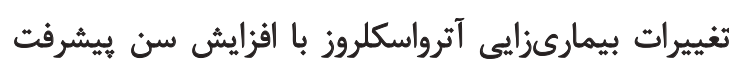

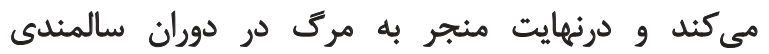

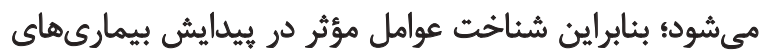

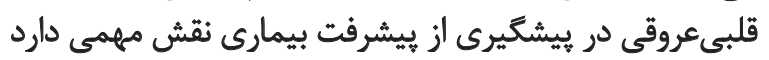

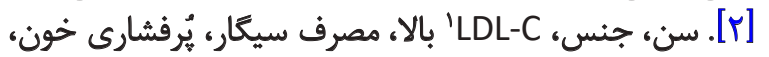


جدول ا. مشخصات عمومى آزمونئىها قبل و بعد از مداخله

\begin{tabular}{|c|c|c|c|c|c|c|}
\hline \multirow{2}{*}{ 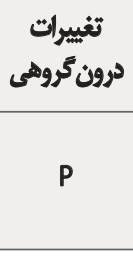 } & \multicolumn{2}{|c|}{ كروه كتترل } & \multirow{2}{*}{ 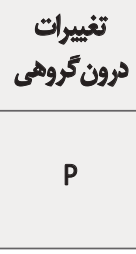 } & \multicolumn{2}{|c|}{ كروه تجيويى } & \multirow{2}{*}{ مشخحات عمومى آز مودنى ها } \\
\hline & استاندارديس أزئمون & 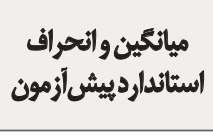 & & 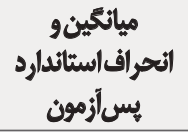 & استانداردييش الثمرون & \\
\hline - & - & EVIRTID/TY & $=$ & $=$ & EVITA $E I T A$ & سن (سال) \\
\hline- & - & $101 / 9 \cdot \pm 8 / \pi 9$ & - & - & $\mid \Delta F / 1 \Delta \pm q / \wedge \Delta$ & قد (سانتىمتر) \\
\hline.$/|r|$ & $8 N / \cdot 1 \pm 1 \% / \cdot 1$ & $Q V / \cdot r \pm r / \Delta r$ & $\cdot 1 \cdot f \lambda$ & $9 r / r \lambda \pm 1 \cdot / 9$ & 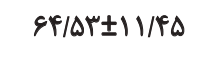 & وزن (كيلوكرم) \\
\hline . /TF. & rq/8. $\pm 8 / 8$ r & rq/rrIE/ND & $\cdot 1 \cdot \Delta 1$ & $r \varepsilon / \& V \pm r / 9 \Lambda$ & $r V / \cdot \Lambda \pm r / / V$ & شاخص توده بدن (kg/m²) \\
\hline. $\mid 4 q 1$ & . & $\cdot / \% 9 १ 9 \pm . / . r V$ & $\cdot 1 \cdot r V$ & $\cdot|A \&| Y \pm * 1 . F 1$ & $. / 918 \cdot \pm \cdot 1 \cdot \mathrm{fp}$ & نسبت كمر به باسن \\
\hline - IAFY & $Y F / \Delta \cdot \pm F / 9 A$ & $Y \Delta / r \Delta \pm V / 9 \Delta$ & $\cdot 1 \cdot A$ & $r V / \cdot . \pm 9 / 1$. & 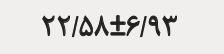 & نيتريكاكسايد (رmol/L) \\
\hline$\cdot / \notin q \Delta$ & IMIAATE-/AD & Ir/AFq土*/qA & .1 .09 & $\mid$ TreTEI/III & $\|M /\| M \pm \| / A \Delta$ & $\begin{array}{c}\text { فشارخون سيستوليك } \\
\text { (ميلى متر جيوه) }\end{array}$ \\
\hline$\cdot M \mid A$ & $A / A \cdot Y \pm \cdot / \Delta \cdot$ & $A N G \pm=/ D F$ & $.1 \cdot . r$ & $N|| \&| \pm \cdot| \Lambda \mid$ & NGFA土*/TS & فشار خون دياستوليك \\
\hline
\end{tabular}

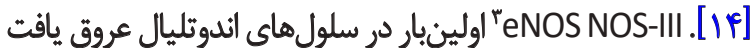
شد. eNOS NOS-III متصل به غشاى سلولى است و باعث انبساط

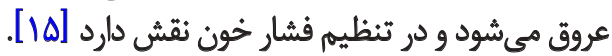
سلول هاى اندوتليال عروقى باتوليد موادفعال كنئده عروق، ماندي

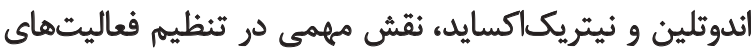

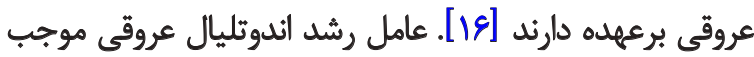

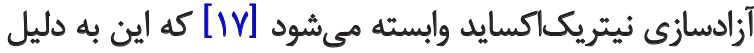

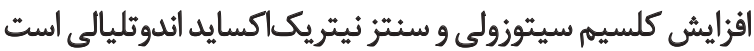

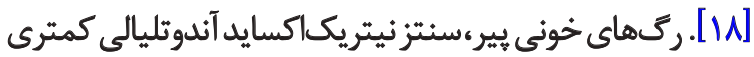

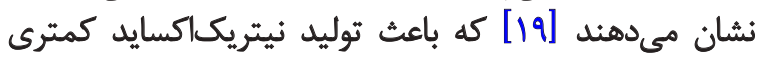

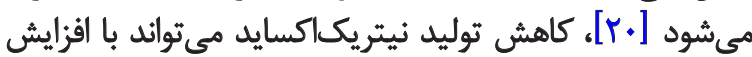

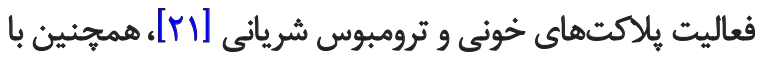

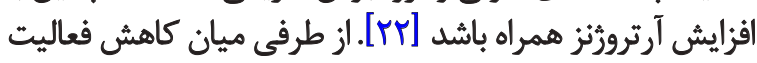

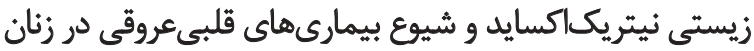

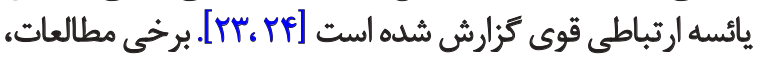

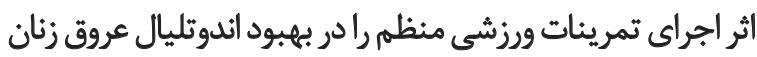

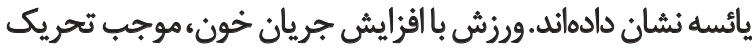
مكانيكى در عروق مىشود و در صورت سالم بودن اندوتليال به به
ايجاد اختالال در عملكرد اندوثليال افراد بير و بركشت عملكرد

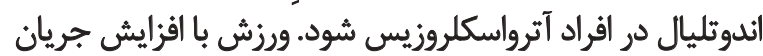

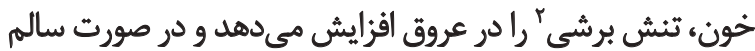

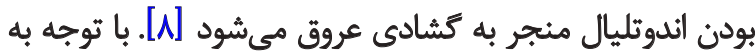

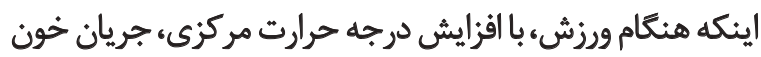

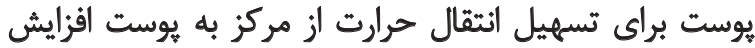

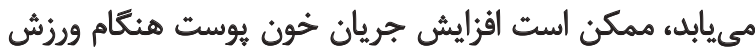

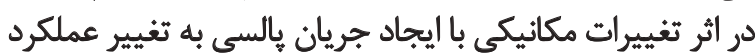

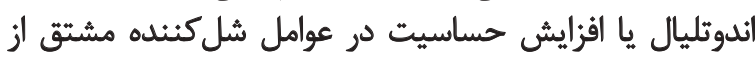

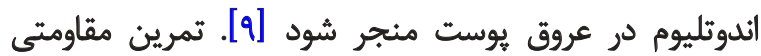

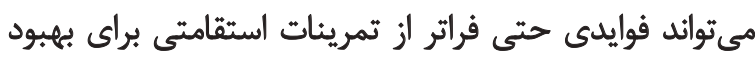

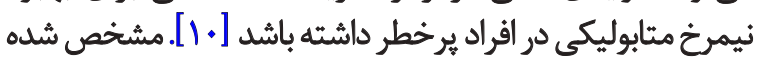

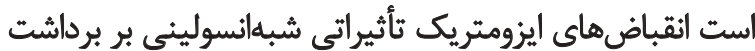
كلوكز در عضله اسكلتى دارند [1/، III]

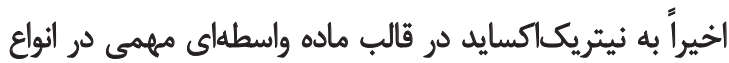

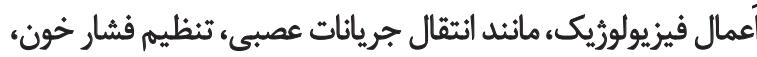

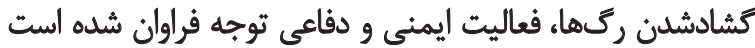

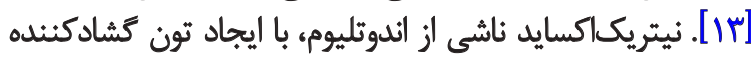

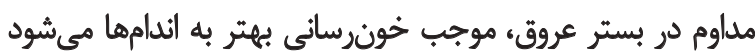

2. Shear Stress 
جدول r. مقايسه اختلاف نيتريكاكسايد و يس آزمون بين كروه تجربى و كنترل

\begin{tabular}{|c|c|c|c|c|c|}
\hline$P$ & $\mathbf{T}$ & اختالك ميانكين & انحراف اسثاندارد & مياتكين & اختالف نيتريكاكسايد \\
\hline \multirow[b]{2}{*}{$+1+1$} & \multirow[b]{2}{*}{-Y/AY. } & \multirow[b]{2}{*}{$\Delta / / \gamma$} & $.18 \Delta$ & epret & تجريى \\
\hline & & &.$/ N$ & $-* N a$ & كتنرل \\
\hline
\end{tabular}

配

BMI= $\frac{\text { قد مجذور (MG) }}{\text { وزن (M) }}$

\section{أندازهيّيرى نيتريكاكسايد}

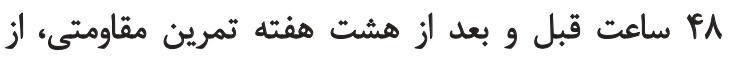

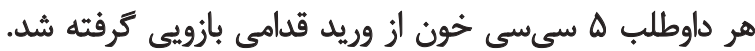

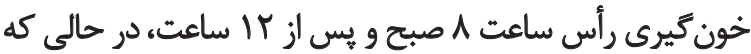

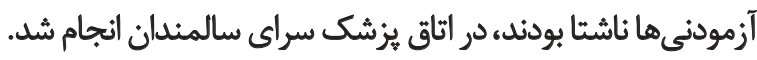

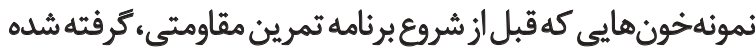

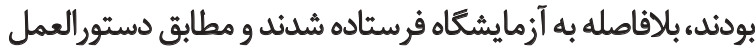

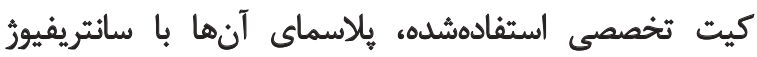

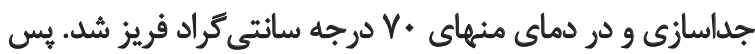

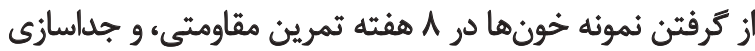

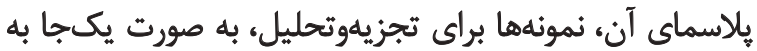

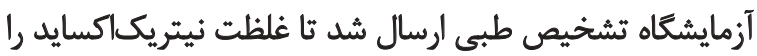

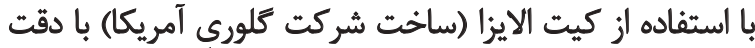

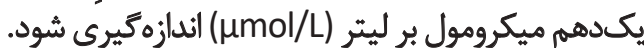

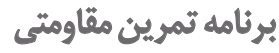

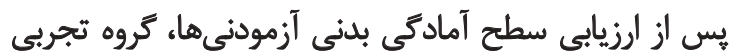

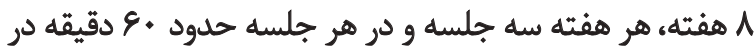

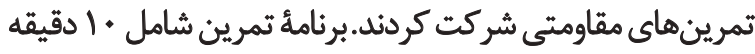

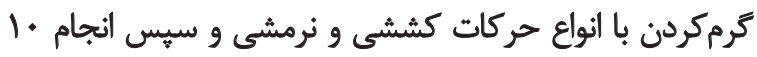

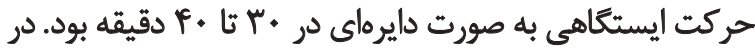

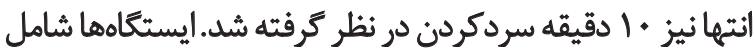

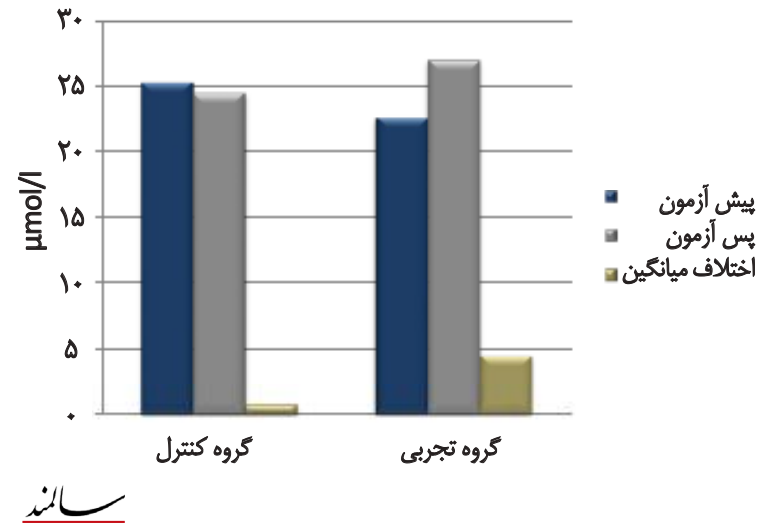

تصوير ا. مقايسه ميائكين نيتريكاكسايد در دو كروه قبل و بعد از مداخله
افزايش توليد و رهايش نيتريكاكسايد منجر مىشود [rه]. هاروى و همكاران در تحقيق خود به نقش درمانى تمرينات

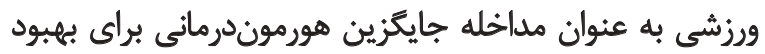

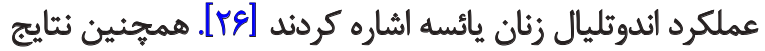

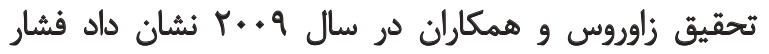

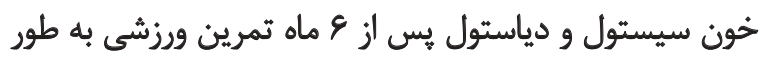

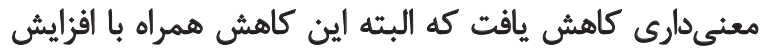

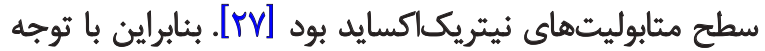

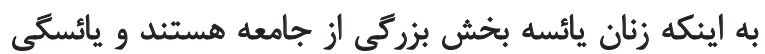

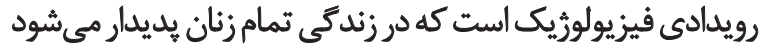

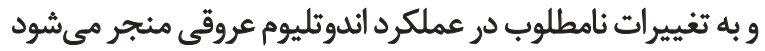

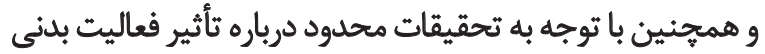

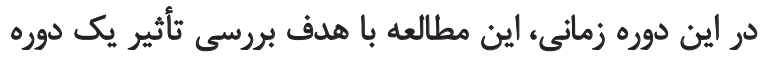
تمرين مقاومتى بر غلظت نيتريكاكسايد زنان سالمند انجام شد.

روش مطالعه

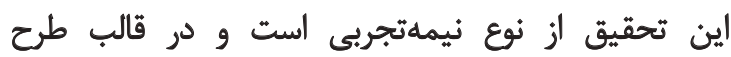

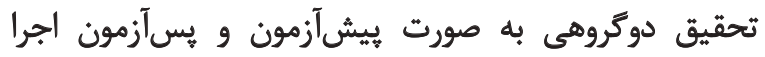

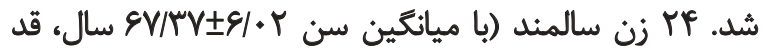

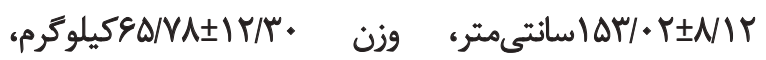

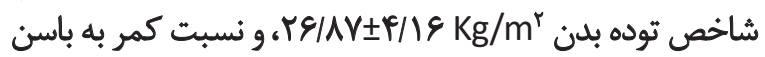


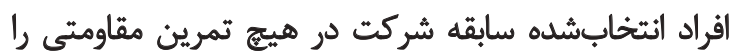

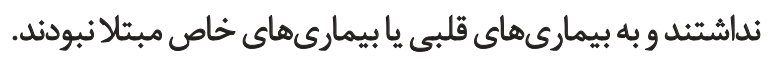

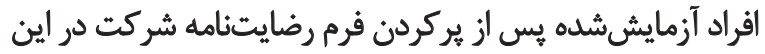

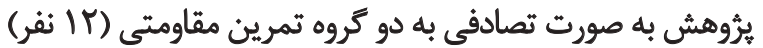

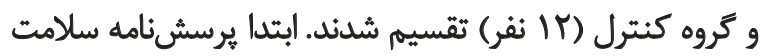

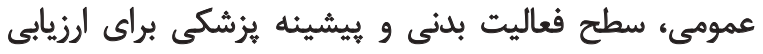

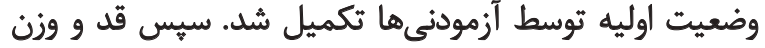

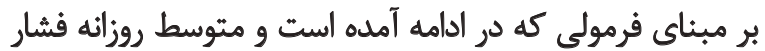

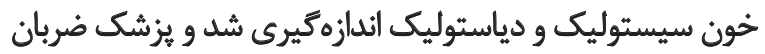

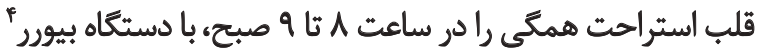

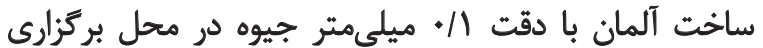
تمرينات اندازهيرى كرد. 
جدول ب. نتايج آزمون همبستكى بيرسون

\begin{tabular}{|c|c|c|c|c|}
\hline فشارخون دياستوليكى & فشار خون ميستوليكى & نيتريكاكسايد & & \\
\hline . / TAY & $\cdot / \Delta A T^{*}$ & 1 & همبستكى ييرسون & \multirow{2}{*}{ نيتريك اكسايد } \\
\hline$+/ 1+*$ & $+1+1 f$ & - & معنى دارى & \\
\hline -/ra4 & 1 &.$/ A M T^{\circ}$ & همبستكى ييرسون & \multirow{2}{*}{ فشار خُون سيستوليك } \\
\hline $1+18$ & - & $+1+15$ & معثى دارى & \\
\hline 1 &.$/$ map &.$/$ TAV & هبمتكى يبير سون & \multirow{2}{*}{ فشار خون دياستوليك } \\
\hline- & $1 \cdot 18$ & $+M+$ & معنى مارى & \\
\hline
\end{tabular}

برخى شاخصها، تفاوت فردى آزمودنى ها از نظر وضعيت روحيى و

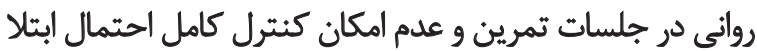
به بيمارى يا آسيب هنكام اجراى تحقيق بود امكان كنترل

Ldî́

مشخصات عمومى آزمودنى ها قبل و بعد از هشت هفته تمرين

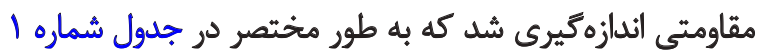

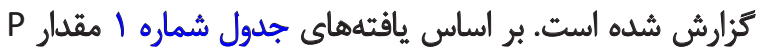

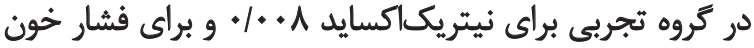

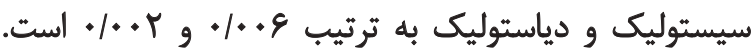

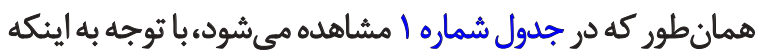

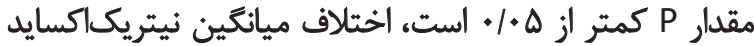

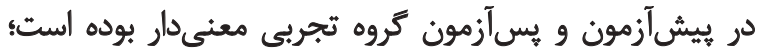

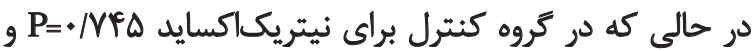

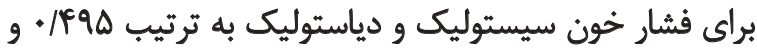

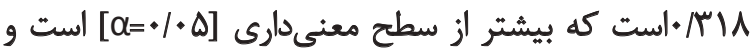

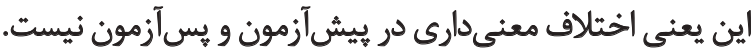

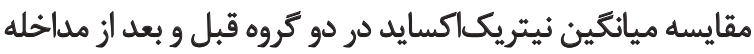

$$
\text { در تصوير شماره آمده است. }
$$

نتايج آزمون t مستقل، براى مقايسه اختلاف يس آزمون

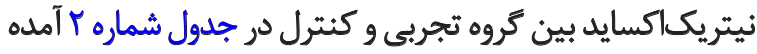

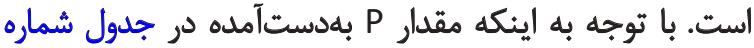

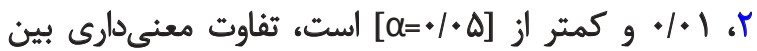

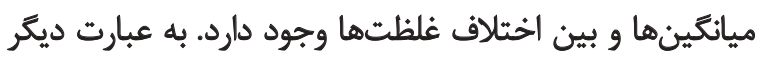

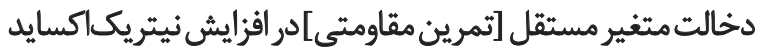

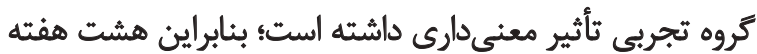

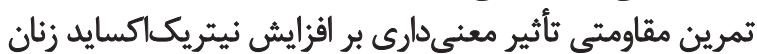

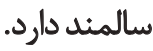

در بررسى رابطه بين متغيرها در جدول شماره با، بين غلظت

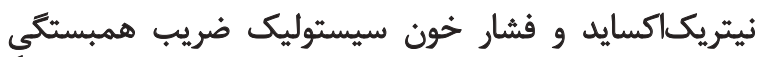

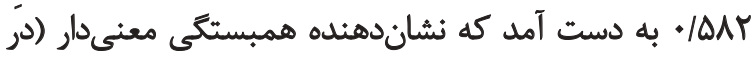

• انتع تمرين مقاومتى (يرس ياء يرس سينه، يرس شانه، جلوبازو،

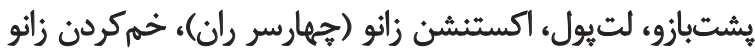

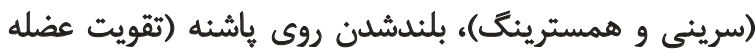

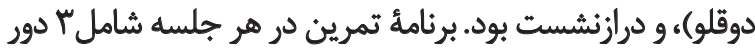

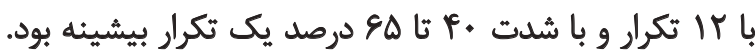

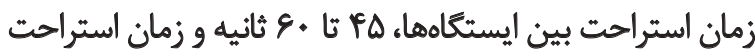

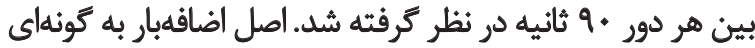

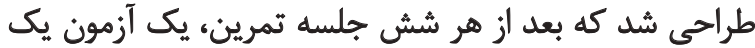

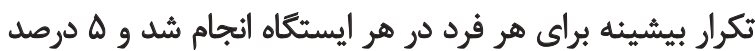

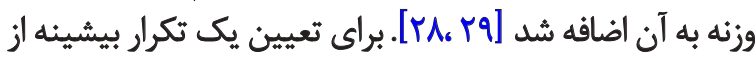

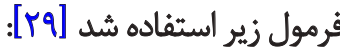

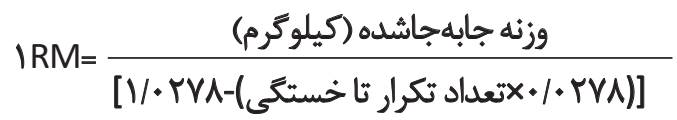

كفتنى است برنامه تمرينى اين تحقيق باتوجه به بر برنامه كادوره

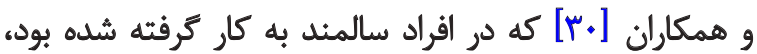

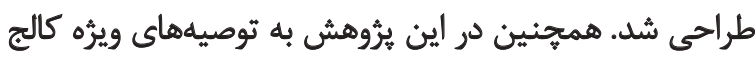

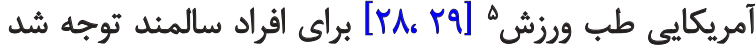

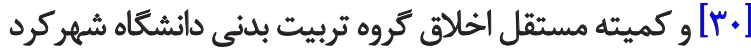

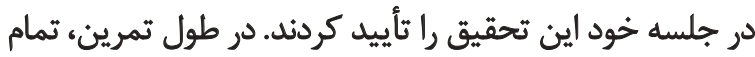

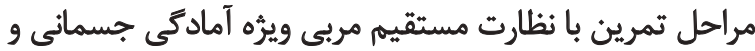

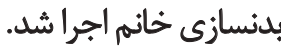

محدوديتهاى اين تحقيق شامل دو دسته بود: محدوديتهاى

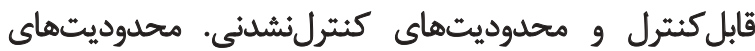

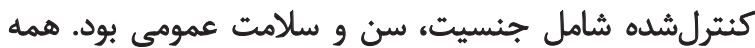

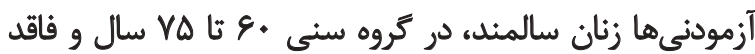

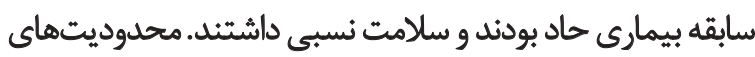

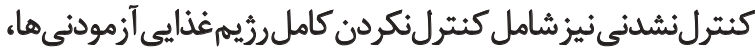

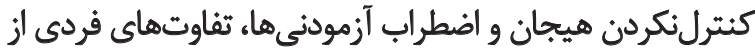

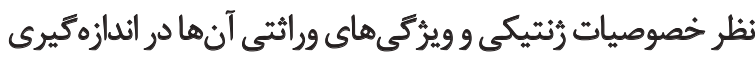

5. ACSM 


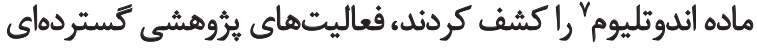

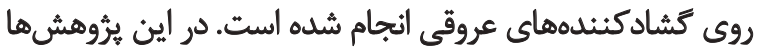

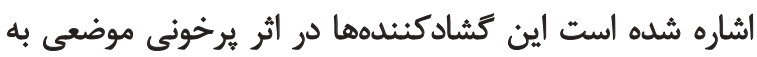

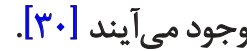

در سال 1919 ميلادى فوركات و همكاران اعلام كردند عامل

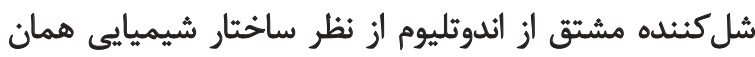

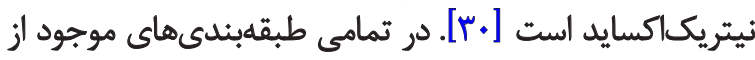

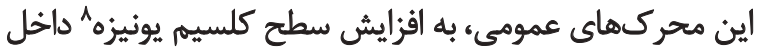

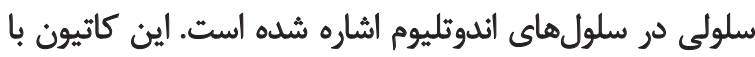

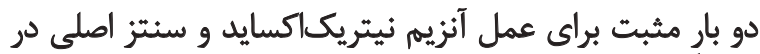

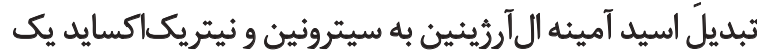
كوفاكتور لازم و ضرورى است. نيتريكاكسايدى كه الز اين واكينش

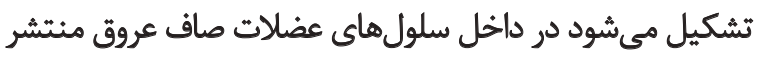

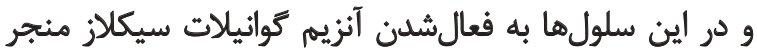

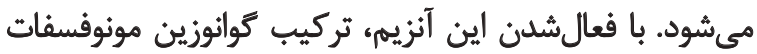

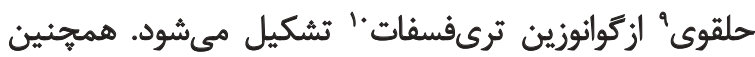

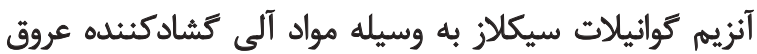

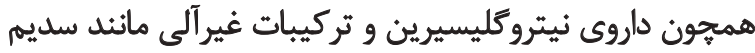

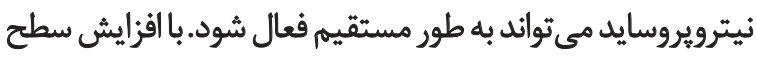

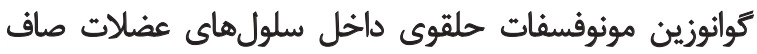

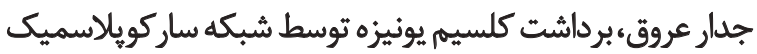

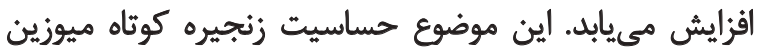

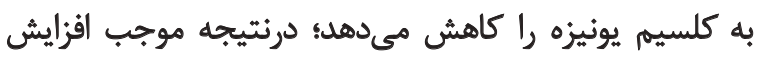

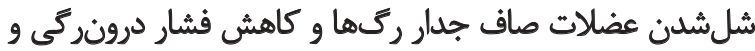

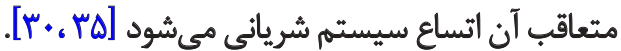

همجنين مطالعات ديكر نشان دادند نيتريكاكسايد در هنكام

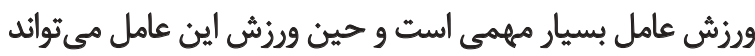

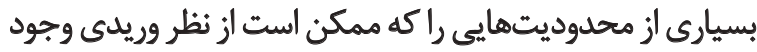

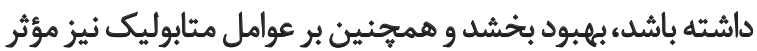

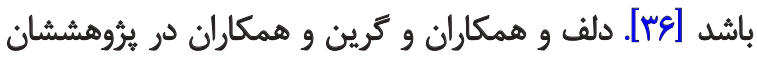

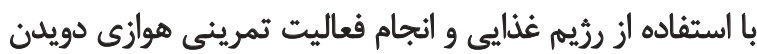

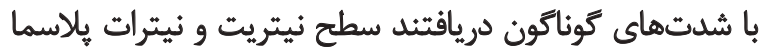

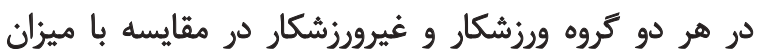

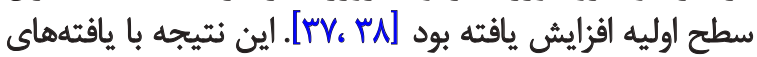

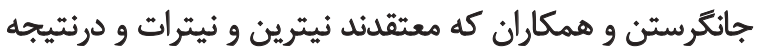

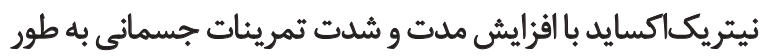

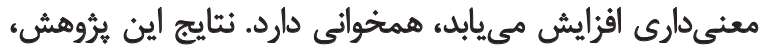

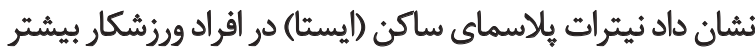

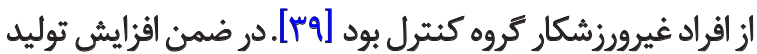

7. EDRF

8. $\mathrm{Ca}^{++}$

9. CGMP

10. GTP
جدول با * مشخص است) و ارتباط مستقيم بين افزايش غلظت

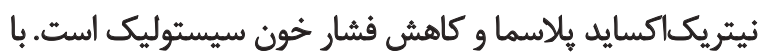

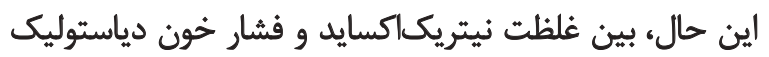

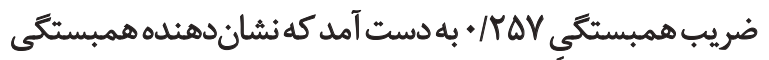

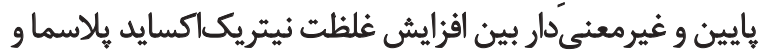
كاهش فشار خون دياستوليك است.

ث)

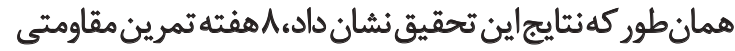

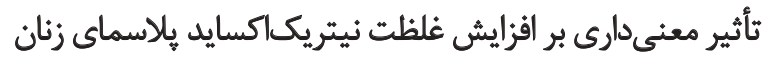

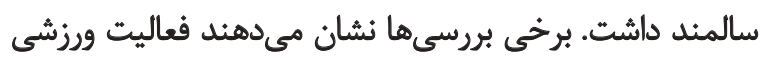

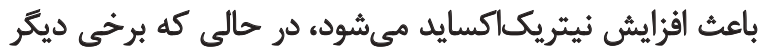

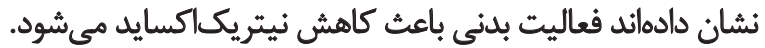

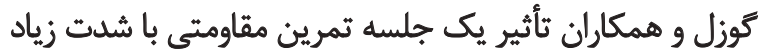

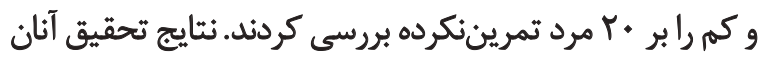

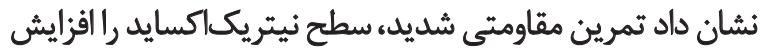

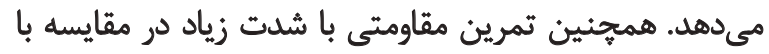

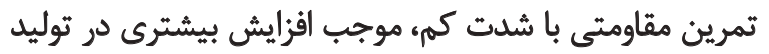

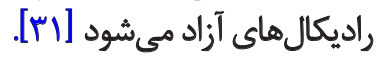

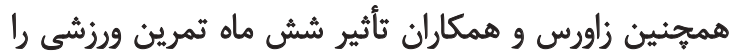

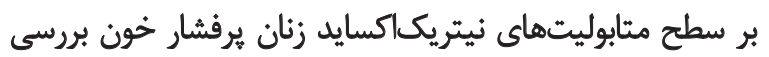

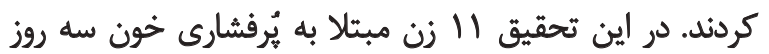

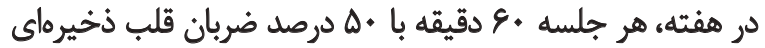

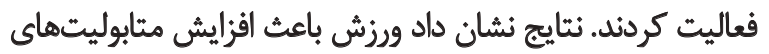

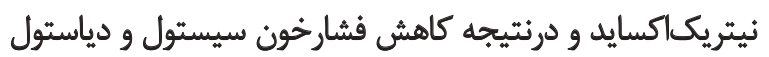

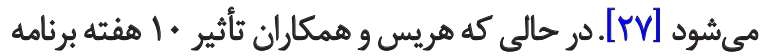

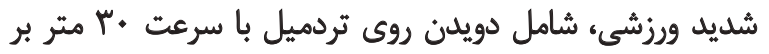

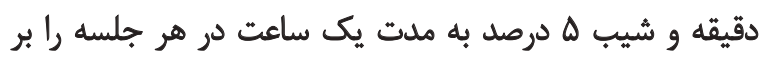

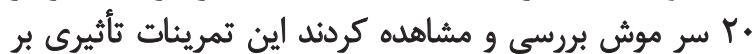

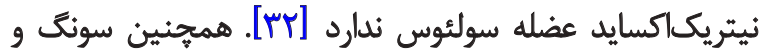

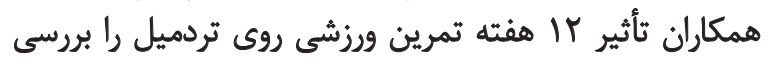

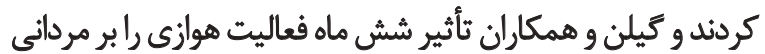

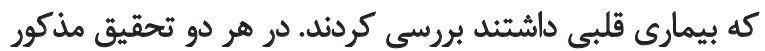

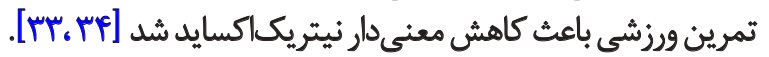

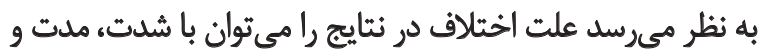

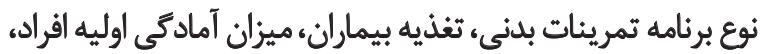
تفاوتهاى فردى و سن آزمودنىها مرتبط دئيات دانست.

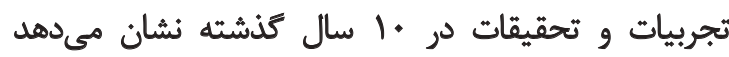

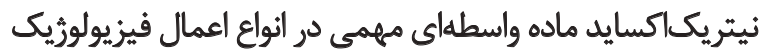

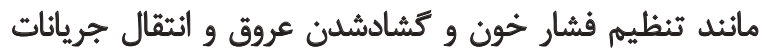

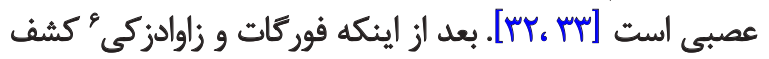




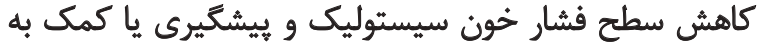

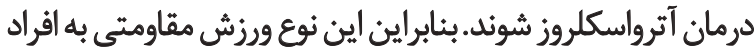

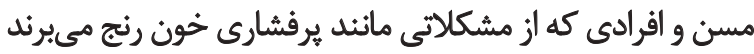
توصيا مي شُون. تشكر وقديرواتي

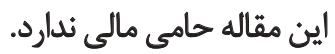

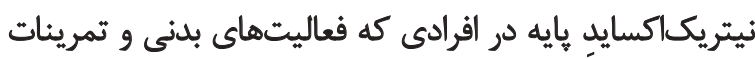

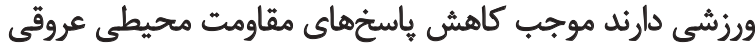

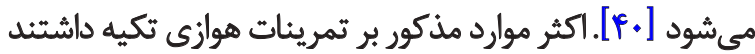
وكمتر ديده شده تأثيرات تمرين مقاومتى سنجيده شورد شود.

نتيجه ديكر برؤهش يمش رون رونشان داد انجام هشت هفته تمرين

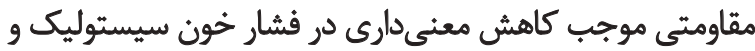

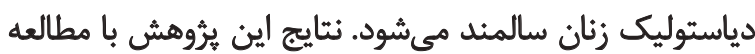

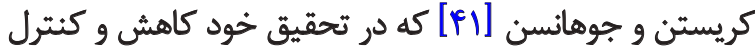

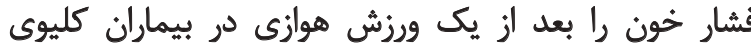

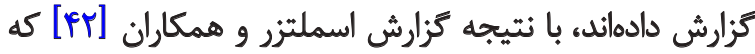

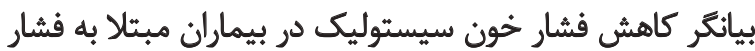

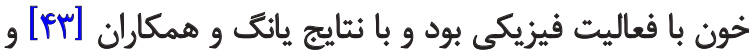

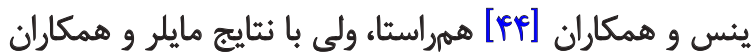

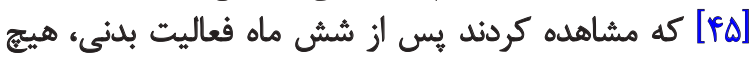

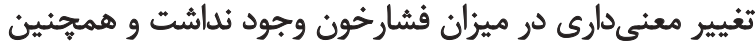

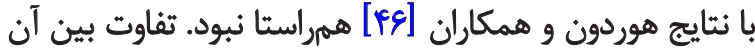

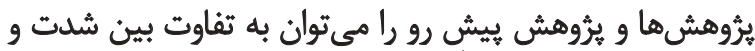

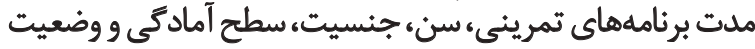

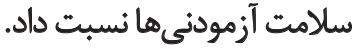

كاهش فشار خون مي تواند به دليل كاهش كاتكولامينهاي

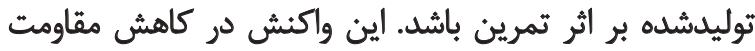

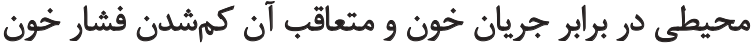

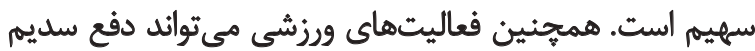

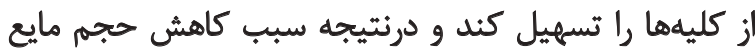

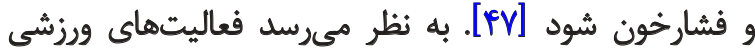

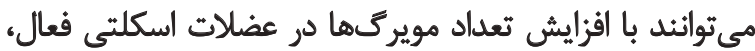

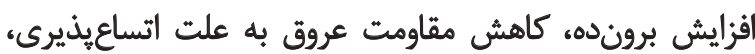

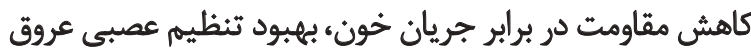

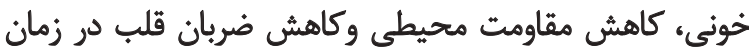

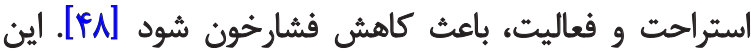

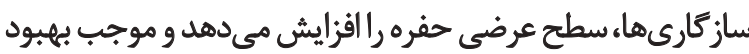

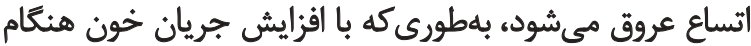

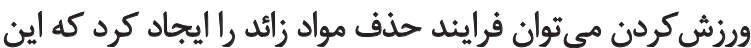

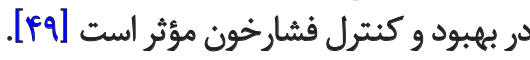

\section{نتيجلكيرى نهايي}

با به طور كلى نتايج اين تحقيق نشان داد تمرينهالى مقاومتى

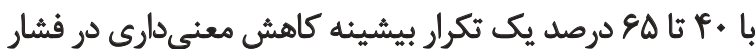

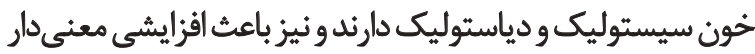

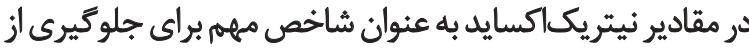

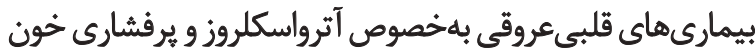

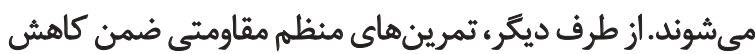

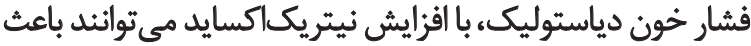




\section{References}

[1] Turk JR, Laughlin MH. Physical activity and atherosclerosis: which animal model? Canadian Journal of Applied Physiology. 2004; 29(5):657-83. doi: 10.1139/h04-042

[2] Lakatta EG, Levy D. Arterial and cardiac aging: Major shareholders in cardiovascular disease enterprises: Part I: Aging arteries: A "set up" for vascular disease. Circulation. 2003; 107(1):139-46. doi: 10.1161/01.cir.0000048892.83521.58

[3] Hagobian TA, Jacobs KA, Subudhi AW, Fattor JA, Rock PB, Muza SR, et al. Cytokine responses at high altitude: Effects of exercise and antioxidants at $4300 \mathrm{~m}$. Medicine \& Science in Sports \& Exercise. 38(2):276-85. doi: 10.1249/01.mss.0000188577.63910.51

[4] Kanaley JA, Sames C, Swisher L, Swick AG, Ploutz-Snyder LL, Steppan CM, et al. Abdominal fat distribution in pre- and postmenopausal women: The impact of physical activity, age, and menopausal status. Metabolism. 2001; 50(8):976-82. doi: 10.1053/ meta.2001.24931

[5] Marques E, Carvalho J, Soares JMC, Marques F, Mota J. Effects of resistance and multicomponent exercise on lipid profiles of older women. Maturitas. 2009; 63(1):84-8. doi: 10.1016/j.maturitas.2009.03.003

[6] Sharman MJ, Volek JS. Weight loss leads to reductions in inflammatory biomarkers after a very-low-carbohydrate diet and a lowfat diet in overweight men. Clinical Science. 2004; 107(4):365-9. doi: $10.1042 / \operatorname{cs} 20040111$

[7] Moncada S, Palmer RM, Higgs EA. Nitric oxide: Physiology, pathophysiology, and pharmacology. Pharmacological Reviews, 1991; 43(2):109-42. PMID: 1852778

[8] Wang JS. Effects of exercise training and detraining on cutaneous microvascular function in man: The regulatory role of endothelium-dependent dilation in skin vasculature. European Journal of Applied Physiology. 2004; 93(4):429-34. doi: 10.1007/s00421-004$1176-4$

[9] Fuchsjager-Mayrl G, Pleiner J, Wiesinger GF, Sieder AE, Quittan M, Nuhr MJ, et al. Exercise training improves vascular endothelial function in patients with type 1 diabetes. Diabetes Care. 2002; 25(10):1795-801. doi: 10.2337/diacare.25.10.1795

[10] Davis PG, Bartoli WP, Durstine JL. Effects of acute exercise intensity on plasma lipids and apolipoproteins in trained runners. Journal of Applied Physiology. 1992; 72(3):914-9. doi: 10.1152/ jappl.1992.72.3.914

[11] Koivisto VA, Eriksson J, Taimela S. Exercise and the metabolic syndrome. Diabetologia. 1997; 40(2):125-35. doi: 10.1007/ s001250050653

[12] Poehlman ET, Dvorak RV, DeNino WF, Brochu M, Ades PA. Effects of resistance training and endurance training on insulin sensitivity in nonobese, young women: A controlled randomized trial. Journal of Clinical Endocrinology \& Metabolism. 2000; 85(7):2463-8. doi: 10.1210/jcem.85.7.6692

[13] Miyauchi T, Masaki T. Pathophysiology of endothelin in the cardiovascular system. Annual Review of Physiology. 1999; 61(1):391-415. doi: 10.1146/annurev.physiol.61.1.391

[14] Donato A, Lesniewski L, Delp M. The effects of aging and exercise training on endothelin-1 vasoconstrictor responses in rat skeletal muscle arterioles. Cardiovascular Research. 2005; 66(2):393401. doi: 10.1016/j.cardiores.2004.10.023
[15] Wennmalm A, Benthin G, Edlund A, Kieler-Jenson N, Lundin S, Petersson AS, et al. Nitric Oxide Synthesis and Metabolism in Man. Annals of the New York Academy of Sciences. 1994; 714(1):158-64. doi: 10.1111/j.1749-6632.1994.tb12040.x

[16] Rubanyi GM, Vanhoutte PM. Oxygen-derived free radicals, endothelium, and responsiveness of vascular smooth muscle. American Journal of Physiology-Heart and Circulatory Physiology. 1986; 250(5):H815-H821. doi: 10.1152/ajpheart.1986.250.5.h815

[17] Ku DD, ZaleskiJK, LiuS, BrockTA. Vascular endothelial growth factor induces EDRF-dependent relaxation in coronary arteries. American Journal of Physiology-Heart and Circulatory Physiology. 1993; 265(2):H586-H592. doi: 10.1152/ajpheart.1993.265.2.h58

[18] Horowitz JR, Rivard A, van der Zee R, Hariawala M, Sheriff DD, Esakof DD, et al. Vascular endothelial growth factor vascular permeability factor produces nitric oxide-dependent hypotension-evidence for a maintenance role in quiescent adult endothelium. Arteriosclerosis, Thrombosis, and Vascular Biology. 1997; 17(11):2793-800. doi: 10.1161/01.atv.17.11.2793

[19] Chou TC, Yen MH, Li CY, Ding YA. Alterations of nitric oxide synthase expression with aging and hypertension in rats. Hypertension. 1998; 31(2):643-8. doi: 10.1161/01.hyp.31.2.643

[20] Taddei S, Virdis A, Ghiadoni L, Salvetti G, Bernini G, Magagna A, et al. Age-related reduction of $\mathrm{NO}$ availability and oxidative stress in humans. Hypertension. 2001; 38(2):274-9. doi: 10.1161/01.hyp.38.2.274

[21] Loscalzo J. Nitric oxide insufficiency, platelet activation, and arterial thrombosis. Circulation Research. 2001; 88(8):756-62. doi: 10.1161/hh0801.089861

[22] Garg UC, Hassid A. Nitric oxide-generating vasodilators and 8-bromo-cyclic guanosine monophosphate inhibit mitogenesis and proliferation of cultured rat vascular smooth muscle cells. Journal of Clinical Investigation. 1989; 83(5):1774-7. doi: 10.1172/ jci114081

[23] Danilov AI, Andersson M, Bavand N, Wiklund NP, Olsson T, Brundin L. Nitric oxide metabolite determinations reveal continuous inflammation in multiple sclerosis. Journal of Neuroimmunology. 2003; 136(1-2):112-8. doi: 10.1016/s0165-5728(02)00464-2

[24] Kahl KG, Zielasek J, Uttenthal LO, Rodrigo J, Toyka KV, Schmidt HHHW. Protective role of the cytokine-inducible isoform of nitric oxide synthase induction and nitrosative stress in experimental autoimmune encephalomyelitis of the DA rat. Journal of Neuroscience Research. 2003; 73(2):198-205. doi: 10.1002/ jnr.10649

[25] Yang AL, Tsai SJ, Jiang MJ, Jen CJ, Chen H. Chronic exercise increases both inducible and endothelial nitric oxide synthase gene expression in endothelial cells of rat aorta. Journal of Biomedical Science. 2002; 9(2):149-55. doi: 10.1007/bf02256026

[26] Kingwell BA. Nitric oxide-mediated metabolic regulation during exercise: effects of training in health and cardiovascular disease. The FASEB Journal. 2000; 14(12):1685-96. doi: 10.1096/ fj.99-0896rev

[27] Zaros PR, Pires CER, Bacci M, Moraes C, Zanesco A. Effect of 6-months of physical exercise on the nitrate/nitrite levels in hypertensive postmenopausal women. BMC Women's Health. 2009; 9(1). doi: 10.1186/1472-6874-9-17

[28] Engelke KA, Halliwill JR, Proctor DN, Dietz NM, Joyner MJ. Contribution of nitric oxide and prostaglandins to reactive hyper- 
emia in the human forearm. Journal of Applied Physiology. 1996; 81(4):1807-14. doi: 10.1152/jappl.1996.81.4.1807

[29] Joyner MJ, Dietz NM. Nitric oxide and vasodilation in human limbs. Journal of Applied Physiology. 1997; 83(6):1785-96. doi: 10.1152/jappl.1997.83.6.1785

[30] Jungersten L, Ambring A, Wall B, Wennmalm Å. Both physical fitness and acute exercise regulate nitric oxide formation in healthy humans. Journal of Applied Physiology. 1997; 82(3):7604. doi: 10.1152/jappl.1997.82.3.760

[31] Guzel NA, Hazar S, Erbas D. Effects of different resistance exercise protocols on nitric oxide, lipid peroxidation and creatine kinase activity in sedentary males. Journal of Sports Science \& Medicine. 2007; 6(4):417-22. PMCID: PMC3794479

[32] Harris MB, Mitchell BM, Sood SG, Webb RC, Venema RC. Increased nitric oxide synthase activity and Hsp90 association in skeletal muscle following chronic exercise. European Journal of Applied Physiology. 2008; 104(5):795-802. doi: 10.1007/s00421008-0833-4

[33] Song W, Kwak HB, Kim JH, Lawler JM. Exercise training modulates the nitric oxide synthase profile in skeletal muscle from old rats. The Journals of Gerontology Series A: Biological Sciences and Medical Sciences. 2009; 64(5):540-49. doi: 10.1093/gerona/glp021

[34] Gielen S, Adams V, Mobius-Winkler S, Linke A, Erbs S, Yu J, et al. Anti-inflammatory effects of exercise training in the skeletal muscle of patients with chronic heart failure. Journal of the American College of Cardiology. 2003; 42(5):861-68. doi: 10.1016/ s0735-1097(03)00848-9

[35] Mcallister RM, Hirai T, Musch TI. ontribution of EndotheliumDerived Nitric Oxide (EDNO) to the skeletal muscle blood flow response to exercise. Medicine \& Science in Sports \& Exercise. 1995; 27(8):1145-51. doi: 10.1249/00005768-199508000-00007

[36] Arsenault BJ, Côté M, Cartier A, Lemieux I, Després JP, Ross $\mathrm{R}$, et al. Effect of exercise training on cardiometabolic risk markers among sedentary, but metabolically healthy overweight or obese post-menopausal women with elevated blood pressure. Atherosclerosis. 2009; 207(2):530-3. doi: 10.1016/j.atherosclerosis.2009.05.009

[37] Delp MD, McAllister RM, Laughlin MH. Exercise training alters endothelium-dependent vasoreactivity of rat abdominal aorta. Journal of Applied Physiology. 1993; 75(3):1354-63. doi: 10.1152/jappl.1993.75.3.1354

[38] Green DJ, Cable NT, Fox C, Rankin JM, Taylor RR. Modification of forearm resistance vessels by exercise training in young men. Journal of Applied Physiology. 1994; 77(4):1829-33. doi: 10.1152/jappl.1994.77.4.1829

[39] Jungersten L, Ambring A, Wall B, Wennmalm Å. Both physical fitness and acute exercise regulate nitric oxide formation in healthy humans. Journal of Applied Physiology. 1997; 82(3):7604. doi: 10.1152/jappl.1997.82.3.760

[40] Duncker D. Nitric oxide contributes to the regulation of vasomotor tone but does not modulate O2-consumption in exercising swine. Cardiovascular Research. 2000; 47(4):738-48. doi: 10.1016/ s0008-6363(00)00143-7

[41] Johansen KL. Exercise and chronic kidney disease: current recommendations. Sports Medicine. 2005; 35(6):485-99. doi: 10.2165/00007256-200535060-00003
[42] Hinkle JL, Cheever KH. Brunner and Suddarth's textbook of medical-surgical nursing [P. Sami, Persian Trans]. Tehran: Boshra; 2000.

[43] Yang K, Bernardo LM, Sereika SM, Conroy MB, Balk J, Burke LE. Utilization of 3-month Yoga program for Adults at High Risk for Type 2 Diabetes. Evidence-Based Complementary and Alternative Medicine. 2011; 2011:1-6. doi: 10.1093/ecam/nep117

[44] Innes KE, Vincent HK. The influence if yoga-based programs on risk profiles in adults with type 2 diabetes mellitus: A systsmatic review. Evidence-Based Complementary and Alternative Medicine. 2007; 4(4):469-86. doi: 10.1093/ecam/nel103

[45] Miller BW, Cress CL, Johnson ME, Nichols DH, Schnitzler MA. Exercise during hemodialysis decreases the use of antihypertensive medications. American Journal of Kidney Diseases. 2002; 39(4):828-33. doi: 10.1053/ajkd.2002.32004

[46] Hordern MD, Cooney LM, Beller EM, Prins JB, Marwick TH, Coombes JS. Determinants of changes in blood glucose response to short-term exercise training in patients with Type 2 diabetes. Clinical Science. 2008; 115(9):273-81. doi: 10.1042/cs20070422

[47] Chen HH. Effects of one-year swimming training on blood pressure and insulin sensitivity in mild hypertensive young patients. The Chinese Journal of Physiology. 2010; 53(4):185-9. doi: 10.4077/cjp.2010.amk042

[48] Andreazzi AE, Scomparin DX, Mesquita FP, Balbo SL, Gravena C, De Oliveira JC, et al. Swimming exercise at weaning improves glycemic control and inhibits the onset of monosodium L-glutamate-obesity in mice. Journal of Endocrinology. 2009; 201(3):3519. doi: 10.1677/joe-08-0312

[49] Johansen KL. Exercise and chronic kidney disease: Current recommendations. Sports Medicine. 2005; 35(6):485-99. doi: $10.2165 / 00007256-200535060-00003$ 
